\title{
An experimental investigation of dual-resonant and non-resonant responses for vortex-induced vibration of a long slender cylinder
}

\author{
WU XiaoDong ${ }^{1,2}$, GE Fei $^{2 *} \&$ HONG YouShi ${ }^{2}$ \\ ${ }^{1}$ College of Mechanics, Taiyuan University of Technology, Taiyuan 030024, China; \\ ${ }^{2}$ State Key Laboratory of Nonlinear Mechanics, Institute of Mechanics, Chinese Academy of Sciences, Beijing 100190, China
}

Received May 27, 2013; accepted June 17, 2013; published online December 30, 2013

\begin{abstract}
Experimental results of the dual-resonant and non-resonant responses are presented for vortex-induced vibrations (VIV) of a long slender cylinder. The cylinder has a diameter of $10 \mathrm{~mm}$ and a length of $3.31 \mathrm{~m}$, giving an aspect ratio of 331 . The cylinder was towed by a carriage with the velocity up to $1.5 \mathrm{~m} / \mathrm{s}$, with the Reynolds number varying from 2500 to 38000 . Three different weights were used to provide the initial tension. Dual resonance means that resonance occurs simultaneously in both the cross-flow (CF) and in-line (IL) directions. The experiments were conducted in two stages. At the first stage, dual-resonant dynamic features of the cylinder subjected to vortex-induced excitation were investigated. The features of CF and IL vibration amplitude, motion orbits, phase angle differences, dominant frequencies and mode order numbers are presented. At the second stage of the experiments, particular emphasis was placed on non-resonant dynamic features. The variation of multi-mode modal displacement amplitudes was investigated in detail.
\end{abstract}

vortex-induced vibrations, long slender cylinder, dual-resonant responses, non-resonant responses

PACS number(s): 47.32.C-, 47.85.Dh, 92.10.Hm

Citation: Wu X D, Ge F, Hong Y S. An experimental investigation of dual-resonant and non-resonant responses for vortex-induced vibration of a long slender cylinder. Sci China-Phys Mech Astron, 2014, 57: 321-329, doi: 10.1007/s11433-013-5279-y

\section{Introduction}

Many kinds of structures exposed to air or water flows may experience vibrations induced by vortex shedding, such as heat exchanger tubes, airplane wings, electric power lines, long span bridges, and underwater risers and cables. While many studies have been focused on vortex-induced vibration (VIV) [1-9], this problem is still far from fully understood, especially for long slender cylinders. One important reason is that testing of such structures needs a large facility and relatively complicated instrumentation, and thus only a few full-scale or large-scale experiments have been carried out.

Model tests [10-17] allow a better understanding of mul-

*Corresponding author (email: gefei@imech.ac.cn) ti-mode behaviors of long slender cylinders. The requirements of these tests consist of large aspect ratio of the cylinder, high sampling rates and careful time synchronization for measurement systems. These, to some extent, restrict the progress in the study of dynamic features of VIV of long slender cylinders. In our experiments, a well-designed test arrangement was used to investigate the dynamic features of a long slender cylinder.

When the vortex shedding frequency is close to the natural frequency of the cylinder, large amplitude resonant response occurs, known as lock-in, which is the most distinct feature of VIV and is observed in numerous laboratory experiments and numerical simulations involving cables, cantilevers, bridge models, and elastically held cylinders $[18,19]$. The cylinder under lock-in often exhibits regular harmonic vibration responses. Generally, lock-in can be 
described as the ability of an elastic structure to control the shedding process in a bandwidth around its resonant frequency. When lock-in occurs, the amplitude of cylinders will increase significantly. However, most of the studies on lock-in are focused on the cases with CF freedom only.

By the end of last century, it had become clear that the vibration in IL direction, though not as large as CF direction, was significant for long slender cylinders. Trim et al. [11] indicated that while IL displacement was less than CF displacement, their modal curvature and vibrating frequency, which dominate fatigue damage, were much larger. A long slender cylinder with a series of natural frequencies may have different modes of vibration excited in CF and IL directions since IL vibration excited by vortex shedding is at distinct frequency from that of CF vibration. Dahl et al. [20] showed that, when the natural frequency ratio (the ratio of natural frequency of IL excited mode to that of CF) equaled the excitation frequency ratio (the ratio of IL excitation frequency to that of $\mathrm{CF}$ ), dual resonance occurred, which meant that the IL and CF motions would resonate simultaneously.

When the vortex shedding frequency is not in the neighborhood of any of the natural frequencies, there is no resonance occurring, the dynamic features will be quite different from those in lock-in regions. We define those regions as non-resonant regions, where the vortex shedding frequency is not in the neighborhood of the natural frequencies. However, little attention has been paid to the dynamic features in these regions until now.

The dynamic features of VIV of long slender structures are important for the safety of marine risers, mooring cables or other similar structures. It deserves more attention from researchers. This paper emphasizes the dual-resonant and non-resonant responses for vortex-induced vibration of a long slender cylinder. First, the dual-resonant results of a test are presented. In our experiments, the dual resonance was studied by analyzing cylinder motion orbits and steady phase angle difference between IL and CF vibrations. Then a summary is given for all tests on dual resonance. Finally, further investigation is conducted on non-resonant dynamic features.

\section{Experimental arrangements}

The experiments were carried out in a towing tank of $29 \mathrm{~m}$ in length, $4.5 \mathrm{~m}$ in width and $4 \mathrm{~m}$ in depth. The tank is equipped with a carriage which tows the model at a given speed, and the overall arrangement of the experiments is shown in Figure 1.

The cylinder model was installed with two universal joints at each end. One universal joint was fixed to a side plate installed at the carriage. The two universal joints connected to the carriage restrict twisting motion of the model, but allow rotating motion of the model. The other universal

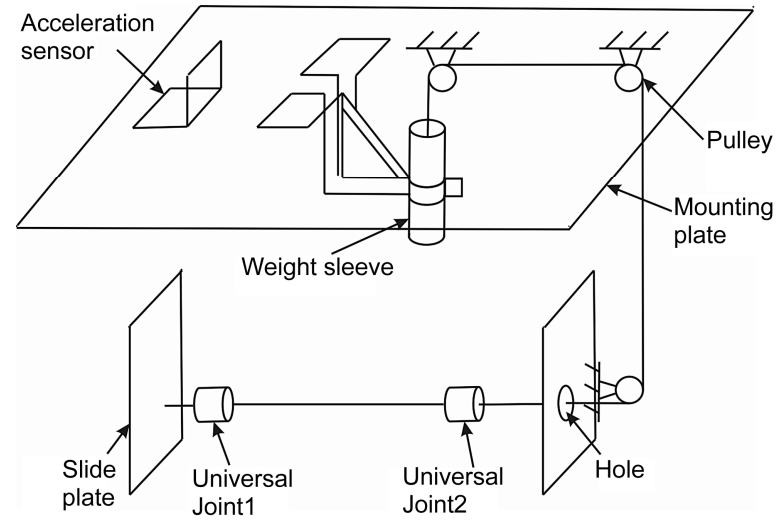

Figure 1 Schematic of the experimental layout.

joint was connected to a weight under the mounting plate through three pulleys by a steel wire. The weight offers an initial tension in the cylinder model. In the experiments, three different weights were used. A ball bearing sleeve was designed to reduce the friction force induced by the sliding of the universal joint, as shown in Figure 2. An acceleration sensor was adopted to monitor the noise induced by the carriage during the experiments.

\subsection{Test model}

In the experiments the model was a $3.31 \mathrm{~m}$ long cylinder with a diameter of $0.01 \mathrm{~m}$, giving an aspect ratio of 331 . Table 1 presents detailed properties of the model. The natural frequencies of the model were obtained through free vibration tests in water. Table 2 shows the first five natural frequencies. It is noted that although the initial tension is different, the last three natural frequencies are similar for all three cases. The reason is that the natural frequencies of higher order modes are less controlled by tension, but dominated by the bending stiffness of the cylinder.

\subsection{Test instrumentation}

It is common that the number of strain sensors must be larger than twice the maximum mode numbers excited in

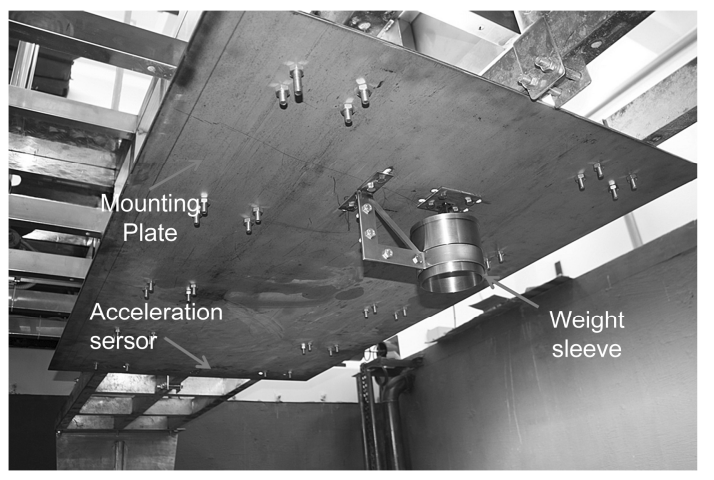

Figure 2 Photo of the side plate and universal joint sleeve. 
Table 1 Model properties

\begin{tabular}{|c|c|c|c|}
\hline & Symbol & Dimension & Value \\
\hline External diameter & $D$ & $\mathrm{~m}$ & 0.01 \\
\hline Length & $L$ & $\mathrm{~m}$ & 3.31 \\
\hline Aspect ratio & $L / D$ & - & 331 \\
\hline Slender stiffness & $E I$ & $\mathrm{Nm}^{2}$ & 26.111 \\
\hline Initial tension & $T$ & $\mathrm{~N}$ & $25,50,75$ \\
\hline Mass ratio & $m^{*}$ & - & 1.867 \\
\hline Axial stiffness & $E A$ & $\mathrm{~N}$ & 2803871 \\
\hline Mass & $m$ & $\mathrm{~kg} / \mathrm{m}$ & 0.1466 \\
\hline Reynolds number & $R e$ & _ & $2500-38000$ \\
\hline
\end{tabular}

Table 2 Natural frequencies, note that the tension provided by weight is nominal tension, actual tension is influenced by friction

\begin{tabular}{cccccc}
\hline Tension $(\mathrm{N})$ & $f_{1}(\mathrm{~Hz})$ & $f_{2}(\mathrm{~Hz})$ & $f_{3}(\mathrm{~Hz})$ & $f_{4}(\mathrm{~Hz})$ \\
\hline 25 & 2 & 6.4 & 13.5 & 23 & 36.0 \\
50 & 2.2 & 6.7 & 13.9 & 23.5 \\
75 & 2.7 & 6.9 & 14.3 & 36.2 & 24.1 \\
\hline
\end{tabular}

$\mathrm{CF}$ and IL directions to make sure that spatial aliasing in computing modal contribution does not occur. Therefore, according to the model material properties, tension and the maximum towing speed, the maximum mode number of the model in our experiments was simulated. The numerical result shows that the max. mode number amounts up to 4 in $\mathrm{CF}$ direction and 5 in IL direction. Thus a total number of 12 strain gauges in each direction were employed, including the extra sensors. The sampling frequency of the strain gauges is $1 \mathrm{kHz}$. The strain gauges in $\mathrm{CF}$ and IL directions share the same space distribution along the model. In the experiments, the fiber optic strain gauges were used, which obtain the strain signal by measuring the wavelength shift of the reflected light.

Displacements were calculated from measured strains by using modal analysis method proposed by Lie and Kaasen [13]. This method is based on the fact that the displacement and strain share the same modal weights. The modal weights are obtained by fitting measured strain to the superposition of sinusoidal mode shape functions that correspond to certain modes. The displacement obtained by this method was in good agreement with the result computed by double spatial integral of the curvature.

\section{Results and discussions}

At the first stage of the experiments, except for the case with tension of $25 \mathrm{~N}$, the model was towed in a speed range from $0.2 \mathrm{~m} / \mathrm{s}$ to $1.5 \mathrm{~m} / \mathrm{s}$, with an increment of $50 \mathrm{~mm} / \mathrm{s}$. It was observed that under the tension of $25 \mathrm{~N}$, when the towing speed was over $1.1 \mathrm{~m} / \mathrm{s}$, large deformation of the model would make the universal joint to slip out of the sleeve. Thus the maximum towing speed for the case of $25 \mathrm{~N}$ ten- sion was set to $1.1 \mathrm{~m} / \mathrm{s}$. At this stage, a total of 328 tests were carried out.

In this section, both the CF and IL amplitudes of strain and displacement, vibrating frequencies, motion orbits, together with phase angle differences are analyzed to study the dual-resonant dynamic characteristics of the model. Firstly, the representative results of one test are given in sect. 3.1. Then all the test results are summarized in sect. 3.2 .

\subsection{Results of one representative test}

Figures 3-8 present typical results of one representative test in which the initial tension is $25 \mathrm{~N}$ and the towing speed is $0.75 \mathrm{~m} / \mathrm{s}$. This test is an example that is used to illustrate the dual resonance.

Figure 3 shows the cross-flow displacement results at some locations along the length of the model. The time series of the cross-flow displacement and an enlargement of a segment are given in the first and second columns, respectively. All the displacement results are normalized with respect to the diameter of the model. After about $5 \mathrm{~s}$, the response seems to reach a steady state. The amplitude of cross-flow displacement is about one diameter of the model. The spectra of each cross-flow displacement are shown in the third column. At each location, only one clear peak is observed at about $11 \mathrm{~Hz}$. The instantaneous cross-flow deflections along the length of the model between 20-20.2 s are given in the last column. The deflections clearly indicate that the cross-flow responses are of the third order mode.

Figure 4 illustrates the in-line displacement results. The time series of displacement, an enlargement of a segment, the spectra and instantaneous in-line deflections are given in each column. Here the mean defections are removed from 


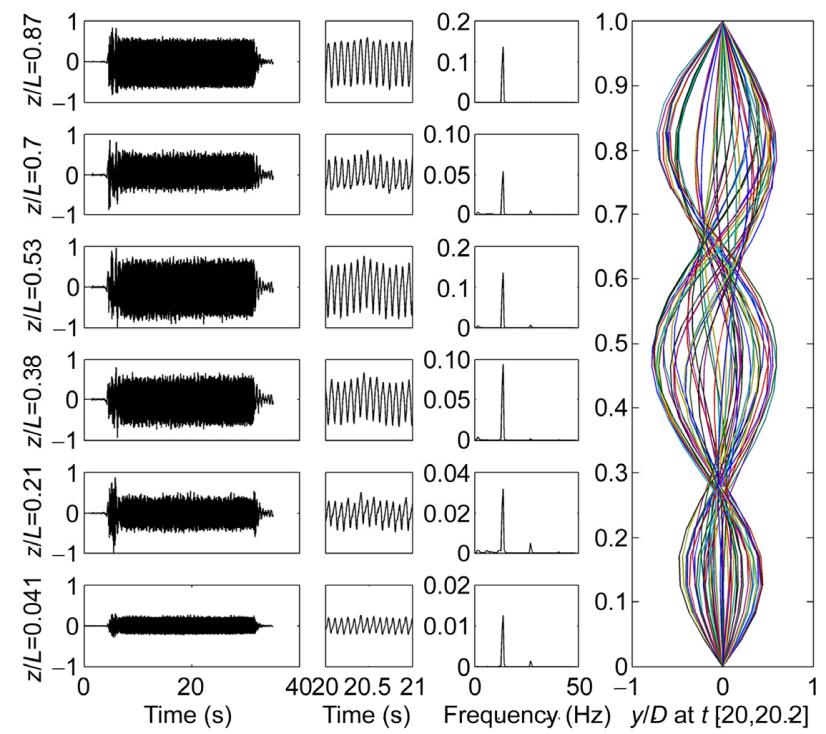

Figure $3 \mathrm{CF}$ displacements along the length of the model for a test with $V=0.75 \mathrm{~m} / \mathrm{s}$ and $T=25 \mathrm{~N}$. First column shows the time series of CF displacements along the length of the model. Second column shows an enlargement of a time segment. Third column shows the spectra of each displacement. Fourth column shows deflections inside a time segment.

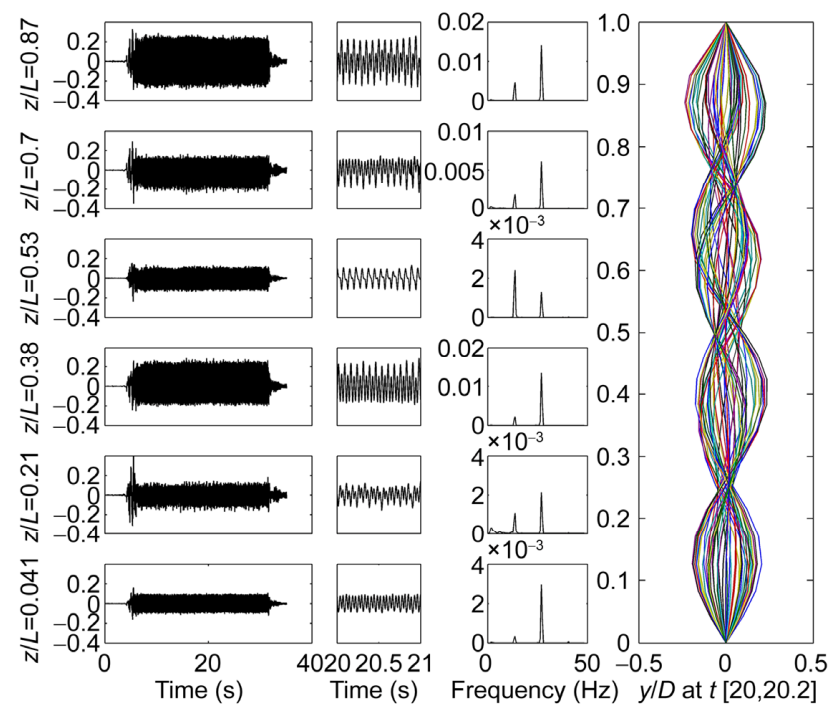

Figure 4 IL displacements along the length of the model for a test with $V=0.75 \mathrm{~m} / \mathrm{s}$ and $T=25 \mathrm{~N}$. First column shows the time series of IL displacements along the length of the model. Second column shows an enlargement of a time segment. Third column shows the spectra of each displacement. Fourth column shows deflections inside a time segment.

the in-line displacements. The in-line exciting frequency is twice that in the cross-flow direction, thus the frequency of in-line displacement is higher than that of cross-flow displacement, as shown in the spectra listed in the third column. The mode order of in-line displacement is the fourth order, higher than that of cross-flow displacement.

Figures 5(a) and 5(b) are surface plots of the spatial spectra distributions of cross-flow and in-line displacements. It can be clearly seen that the dominant frequencies of
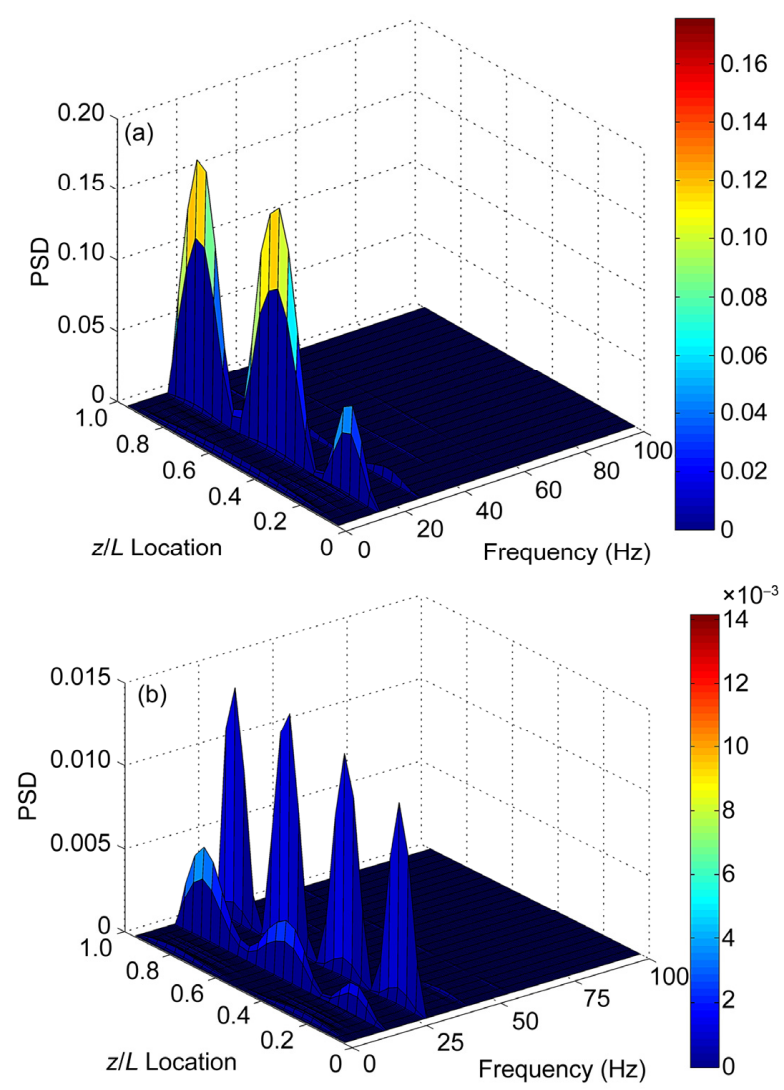

Figure 5 Surface plots of the spatial spectra distributions of (a) crossflow and (b) in-line displacements for a test: $V=0.75 \mathrm{~m} / \mathrm{s}, T=25 \mathrm{~N}$.

cross-flow and in-line displacements are about 13 and 26 $\mathrm{Hz}$, respectively. The spatial spectrum distributions of cross-flow and in-line displacements clearly indicate that the cross-flow and in-line responses are of the third and fourth order modes, respectively. This is consistent with the mode order obtained through mode deflections shown in Figures 3 and 4.

The motion orbits along the length of the model between 20-20.3 s are shown in Figure 6. The motion orbit shapes vary significantly along the length of the model. The orbit shape appears as a clear figure of Arabic numeral eight at the location of $z / L=0.041$ while it becomes very messy at the location of $z / L=0.62$. The motion orbit shape has an influence on vortex shedding pattern, and therefore on the lift and drag forces [20]. The significant variation of the motion orbit shape along the model length indicates variation of the lift and drag forces even in a uniform flow.

Vandiver et al. [21] suggested that the motion orbit shape was controlled by the phase angle difference between the cross-flow and in-line motions. The motion orbit shapes vary significantly along the length of the model. Therefore the phase angle differences along the length of the model are expected to change significantly. The phase angle differences will be investigated in detail below.

For the purpose of obtaining the instantaneous phase angle of cross-flow or in-line displacement, the measured data 

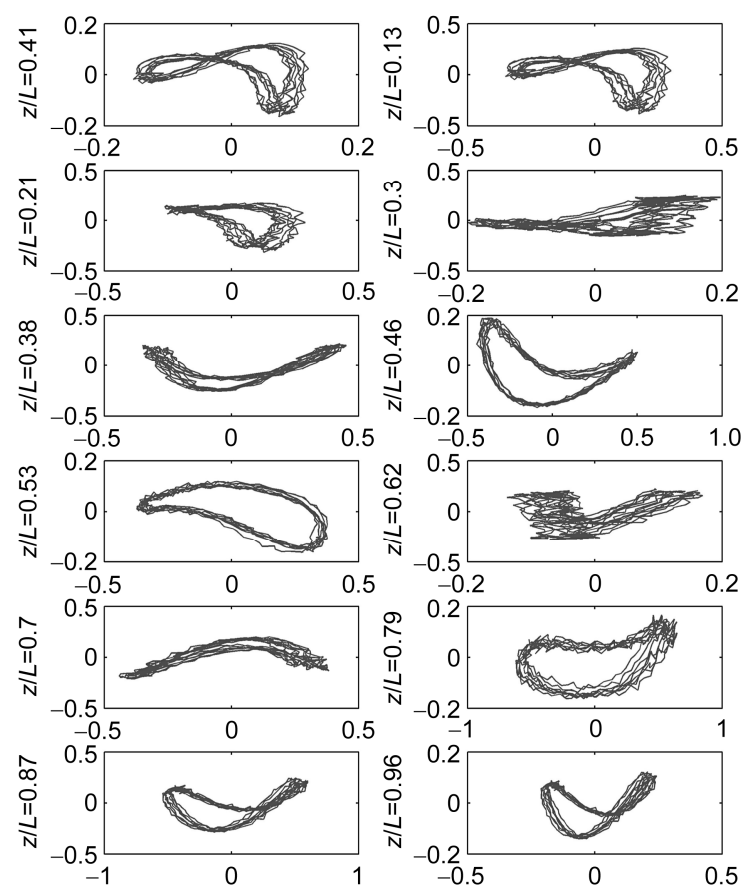

Figure 6 Motion orbits along the length of the model for a test with $V=$ $0.75 \mathrm{~m} / \mathrm{s}$ and $T=25 \mathrm{~N}$.

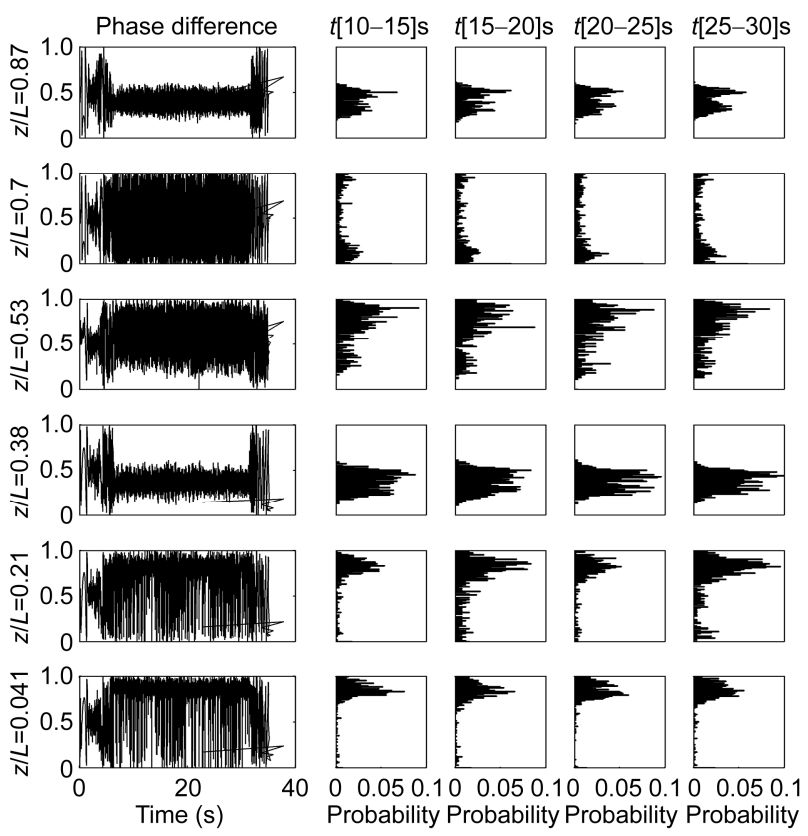

Figure 7 Phase angle differences along the length of the model for a test with $V=0.75 \mathrm{~m} / \mathrm{s}$ and $T=25 \mathrm{~N}$. First column shows time series of phase angle differences for the whole run. The other four columns the probability distributions of the phase angle difference along the length of the model at different time intervals.

have to be translated into analytical signals using time frequency analysis such as Hilbert transform [22]:

$$
\xi\left(z_{i}, t_{j}\right)=s\left(z_{i}, t_{j}\right)+\mathrm{i} s_{H}\left(z_{i}, t_{j}\right)=A\left(z_{i}, t_{j}\right) \mathrm{e}^{\mathrm{i} \phi\left(z_{i}, t_{j}\right)},
$$

where $s\left(z_{i}, t_{j}\right)$ is the cross-flow or in-line displacement at height $z_{i}$ and instant $t_{j}, s_{H}\left(z_{i}, t_{j}\right)$ is the Hilbert transform of $s\left(z_{i}, t_{j}\right)$ and $A\left(z_{i}, t_{j}\right)$ and $\phi\left(z_{i}, t_{j}\right)$ are the instantaneous amplitude and phase angle, respectively. The cross-flow or in-line instantaneous frequency is defined by the following equation:

$$
\Omega\left(z_{i}, t_{j}\right)=\frac{\phi\left(z_{i}, t_{j}\right)-\phi\left(z_{i}, t_{j-1}\right)}{\Delta T},
$$

where $\Delta T$ is the sampling instant and $\Omega\left(z_{i}, t_{j}\right)$ is the instantaneous frequency at height $z_{i}$ and instant $t_{j}$. The definition of the phase angle difference of two periodic signals is given as [23]:

$$
\phi_{I L, C F}\left(z_{i}, t_{j}\right)=n \phi_{I L}\left(z_{i}, t_{j}\right)-m \phi_{C F}\left(z_{i}, t_{j}\right)
$$

where $\phi_{I L, C F}\left(z_{i}, t_{j}\right)$ is the phase angle difference, $n$ and $m$ are integers, relating to the cross-flow and in-line instantaneous frequencies by the following equation:

$$
\frac{n}{m}=\frac{\Omega_{I L}\left(z_{i}, t_{j}\right)}{\Omega_{C F}\left(z_{i}, t_{j}\right)} .
$$

For narrow banded signals, the phase angle difference exhibits a plateau in its variation curve versus time. However, the measured data include noise, and for long slender cylinder the response is excited in more than one mode. Hence, the computed phase angle difference may deviate from its real value. Figure 7 shows the phase angle differences at different locations along the model for the same test. The phase angle differences are normalized by $2 \pi$. The first column shows the time series of the phase angle differences, which indicates that the phase angle differences at each location fluctuate around a steady value as time progresses, except for the locations of $z / L=0.53$ and 0.7 . Other columns in Figure 7 show the probability distributions of the phase angle difference along the length of the model at different time intervals. It is shown that there is a peak at each probability distribution diagram, indicating the existence of steady phase angle differences. In this paper, a statistical method [24] is adopted to compute the steady phase angle differences. It is supposed to find the best Gaussian fit to the computed phase angle differences by the following equation:

$$
\gamma\left(z_{i}, t_{j}\right)=\frac{1}{\sigma \sqrt{2 \pi}} \mathrm{e}^{-\frac{1}{2}\left[\phi_{L, C F}\left(z_{i}, t_{j}\right)-\mu / \sigma\right]^{2}},
$$

where $\mu$ is the mean value and $\sigma$ is the standard deviation of the distribution. Figure 8 shows the fitted phase angle differences at different locations. The phase angle difference 


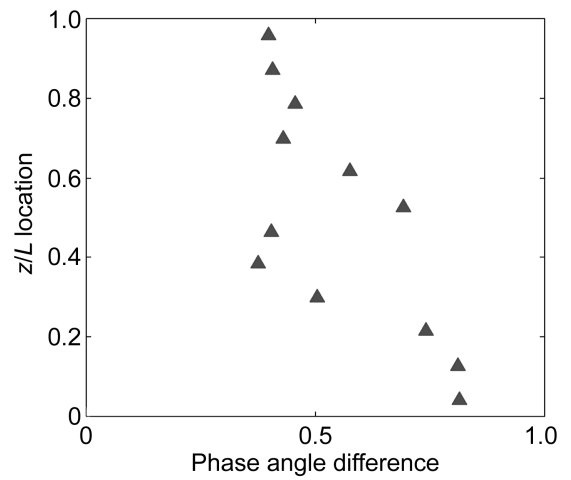

Figure 8 Fitted phase angle differences along the length of the model for a test with $V=0.75 \mathrm{~m} / \mathrm{s}$ and $T=25 \mathrm{~N}$.

varies significantly along the length of the model as expected.

\subsection{Summary of all text results in the first stage}

In this section, the maximum RMS (root mean square) amplitudes of CF and IL displacements and strains, mode order number and frequency of the strain are presented as functions of the towing speed. The peak frequency of strain at the midpoint of the model is adopted as the dominant frequency.

Figure 9 shows how the maximum RMS amplitude of displacement varies with the towing speed. The cross-flow RMS amplitude keeps increasing until it reaches a peak of about $0.63 D$ at about $0.3 \mathrm{~m} / \mathrm{s}$, and then decreases. Three peaks are observed within the towing speed range. The in-line maximum RMS amplitude increases with the towing speed until $0.6 \mathrm{~m} / \mathrm{s}$, and then remains almost the same. Besides, the in-line RMS displacement amplitude is considerably smaller than the cross-flow one.

Figure 10 suggests that the cross-flow and in-line maxi

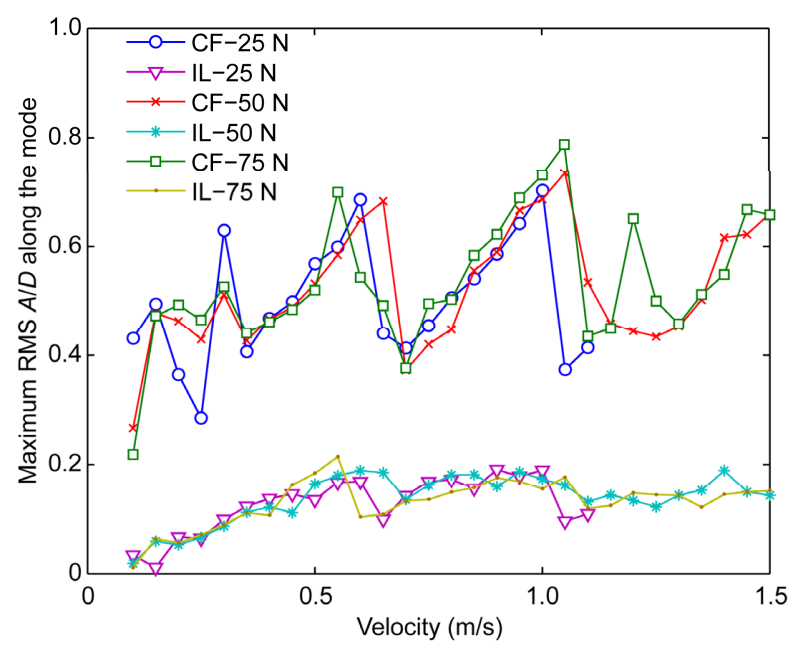

Figure 9 (Color online) Maximum RMS displacement amplitude along the length.

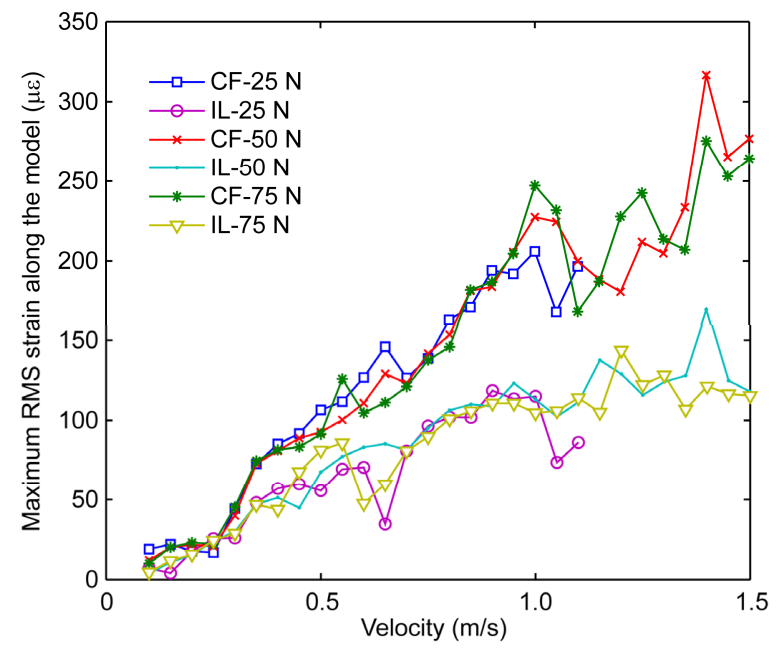

Figure 10 (Color online) Maximum RMS strain amplitude along the length.

mum RMS amplitudes of strain increase progressively with the towing speed for all three cases with different tensions. It is noted that the in-line strain is not much smaller than the cross-flow strain.

The excited mode at each location may be different. The dominant mode order number along the length is adopted as the mode order number in Figure 11. It seems from the figure that the mode order number increases with the towing speed for all three different tension cases. The maximum excited mode order numbers in cross-flow and in-line directions are 4 and 5 , respectively.

Figure 12 presents the strain frequencies varying with the towing speed for three different tension cases. The frequencies show linearly, slowly increasing trends over the whole towing speed range, except that they rise abruptly at those towing speeds at which the mode order number changes.
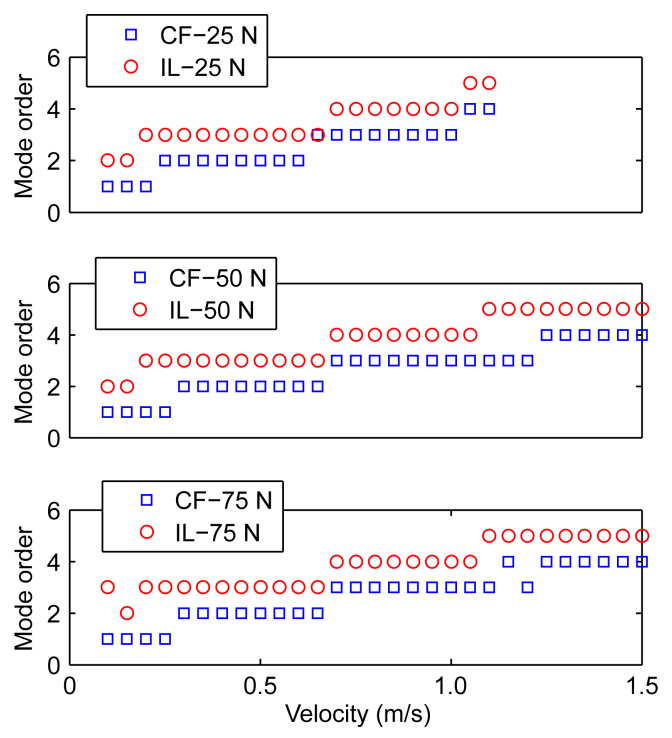

Figure 11 (Color online) Mode order numbers versus the towing speed. 


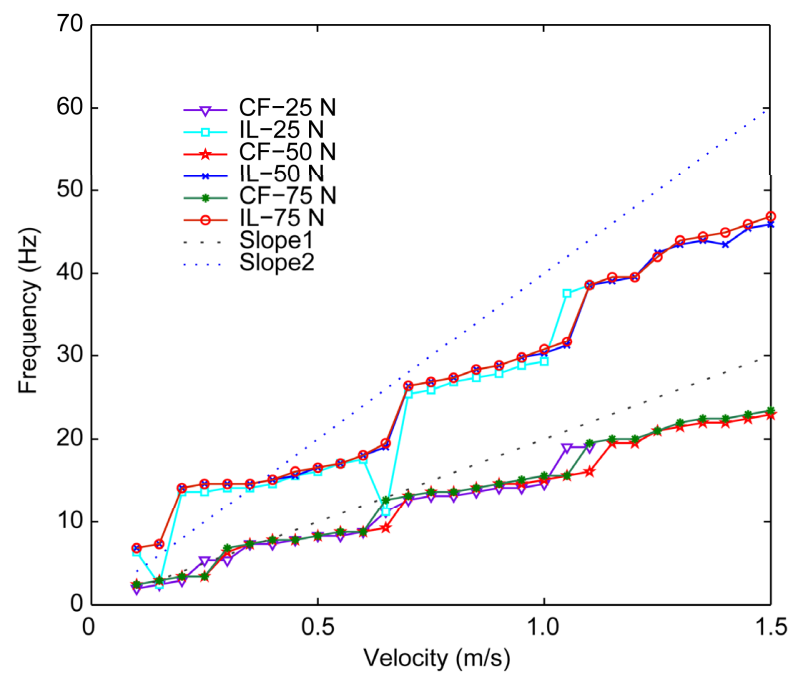

Figure 12 (Color online) Strain frequencies versus the towing speed.

The lower straight line is the Strouhal frequency, based on the towing speed and the Strouhal number of 0.17. The upper straight line is twice the Strouhal frequency. It is observed that the difference between the cross-flow strain frequency and the Strouhal frequency increases with the towing speed, and the difference between the in-line strain frequency and the double of the Strouhal frequency behaves the same way.

\subsection{Investigation of dynamic characteristics in non- resonant regions}

The speed range is roughly divided into resonant regions and non-resonant regions, based on whether the vortex shedding frequency is close to one of the natural frequencies. The vortex shedding frequency in the resonant regions is close to one of the natural frequencies, and thus lock-in is most likely to occur. The vortex shedding frequency in non-resonant regions is not close to any of the natural frequencies, and thus the vibration of the model in these regions is unlikely to resonate with the vortex shedding. Table 3 presents the resonant regions and non-resonant regions. As shown in Figures 9-12, in the non-resonant regions, the displacement amplitude is considerably smaller than that in the resonant regions, the mode order number changes, and the frequency of strain experiences sudden rises. However, the variation of strain amplitude in the non-resonant regions shares the same increasing trend as in the resonant regions.

Figure 13 presents the IL displacements of one representative test in the non-resonant regions, in which the initial tension is $25 \mathrm{~N}$ and the towing speed is $0.66 \mathrm{~m} / \mathrm{s}$. It is noted that the time series of IL displacements are modulated with time, exhibiting several modal contents, except for those strongly periodic at the locations of $z / L=0.041$ and 0.21 . The spectra of IL displacements at most of the locations have more than one peak, indicating that more than
Table 3 Resonant regions and non-resonant regions, note that the division of the speed range is based on the variation of the amplitudes of displacement and strain and it is relatively rough

\begin{tabular}{cc}
\hline Region & Speed range $(\mathrm{m} / \mathrm{s})$ \\
\hline Resonant region 1 & $0.1-0.2$ \\
Non-resonant region 1 & $0.2-0.3$ \\
Resonant region 2 & $0.3-0.55$ \\
Non-resonant region 2 & $0.55-0.75$ \\
Resonant region 3 & $0.75-1.1$ \\
Non-resonant region 3 & $1.1-1.3$ \\
Resonant region 4 & $1.3-1.5$ \\
\hline
\end{tabular}

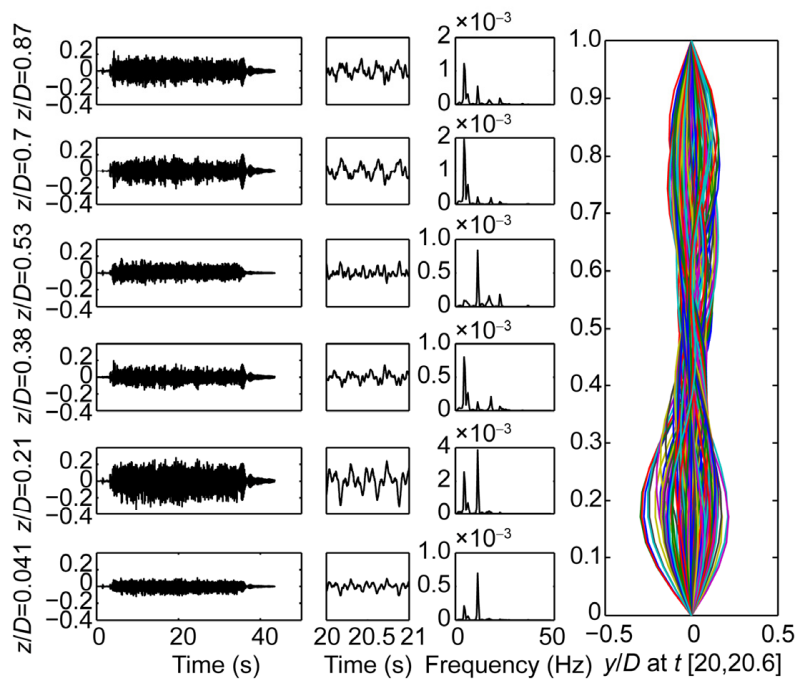

Figure 13 (Color online) IL displacements along the length of the model for a test with $V=0.66 \mathrm{~m} / \mathrm{s}$ and $T=25 \mathrm{~N}$. First column shows the time series of IL displacements along the length of the model. Second column shows an enlargement of a time segment. Third column shows the spectra of each displacement. Fourth column shows deflections inside a time segment.

one mode participate in the vibrations of the model. The fourth column also shows that the vibration is not of a certain mode, but a superposition of several modes. The time series of IL modal displacement from 13-16 s at the location of $z / L=0.041$ are shown in Figure 14. Each time series represents the displacement of one mode. Their sum, weighted by the value of the respective mode shapes at each location, equals the local displacement there. It is indicated that each modal displacement has almost the same amplitude except for mode 5. Figure 15 presents the motion orbits of one test in the non-resonant regions. The motion orbit shapes are quite messy at all locations along the model. Therefore, it is concluded that there are no steady phase angle differences along the model in this test.

The second stage of the experiments was to investigate the dynamic characteristics in non-resonant regions in more detail. The towing speed covered the range of $5.5 \mathrm{~m} / \mathrm{s}$ to 7.5 $\mathrm{m} / \mathrm{s}$, with a refined increment of $5 \mathrm{~mm} / \mathrm{s}$. A total of 275 cases were tested in this stage. Figures 16(a) and 16(b) show how the RMS values of CF and IL modal displacement vary with the towing speed for the initial tension of $75 \mathrm{~N}$. The 


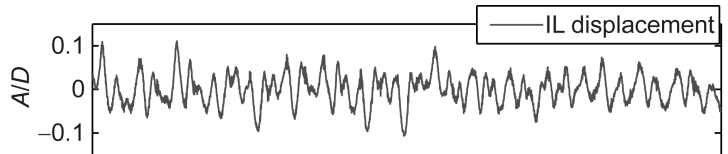
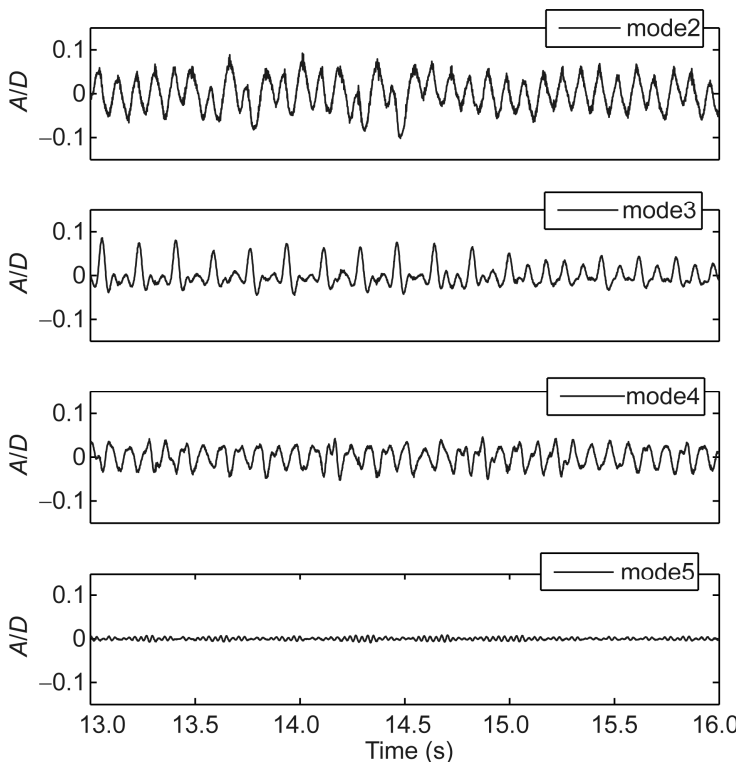

Figure 14 Time series of IL modal displacements at the location of $z / L=$ 0.041 for a test with $V=0.66 \mathrm{~m} / \mathrm{s}$ and $T=25 \mathrm{~N}$.
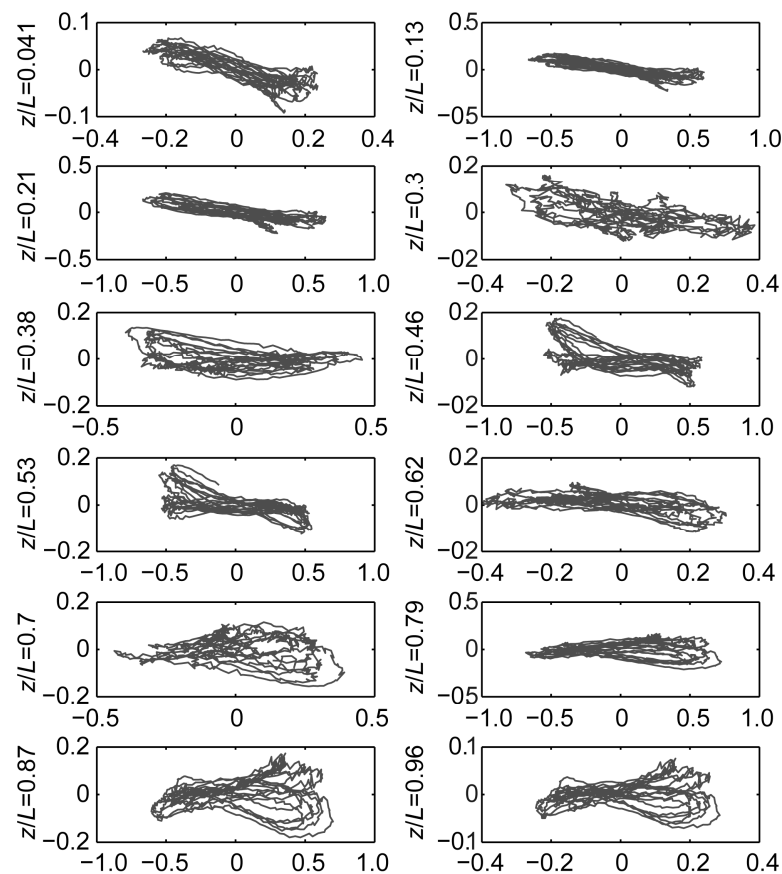

Figure 15 Motion orbits along the length of the model for a test in nonresonant regions: $V=0.66 \mathrm{~m} / \mathrm{s}, T=25 \mathrm{~N}$.

RMS value of individual modal displacement exhibits a peak in their respective excited speed ranges. These excited ranges fall in the corresponding resonant regions. In the resonant regions, the amplitude of one modal displacement is significantly larger than that of other modal displace- ments. However, in non-resonant regions, no single mode is found to dominate the displacement amplitude. Figures 16(a) and 16(b) also show that, in non-resonant regions, modal displacements share almost the same amplitudes. These modes compete with each other to dominate the vibration of model. Table 4 gives these competing modes in different non-resonant regions, respectively.

\section{Conclusions}

In this paper, the dual-resonant and non-resonant responses are investigated for vortex-induced vibration of a long
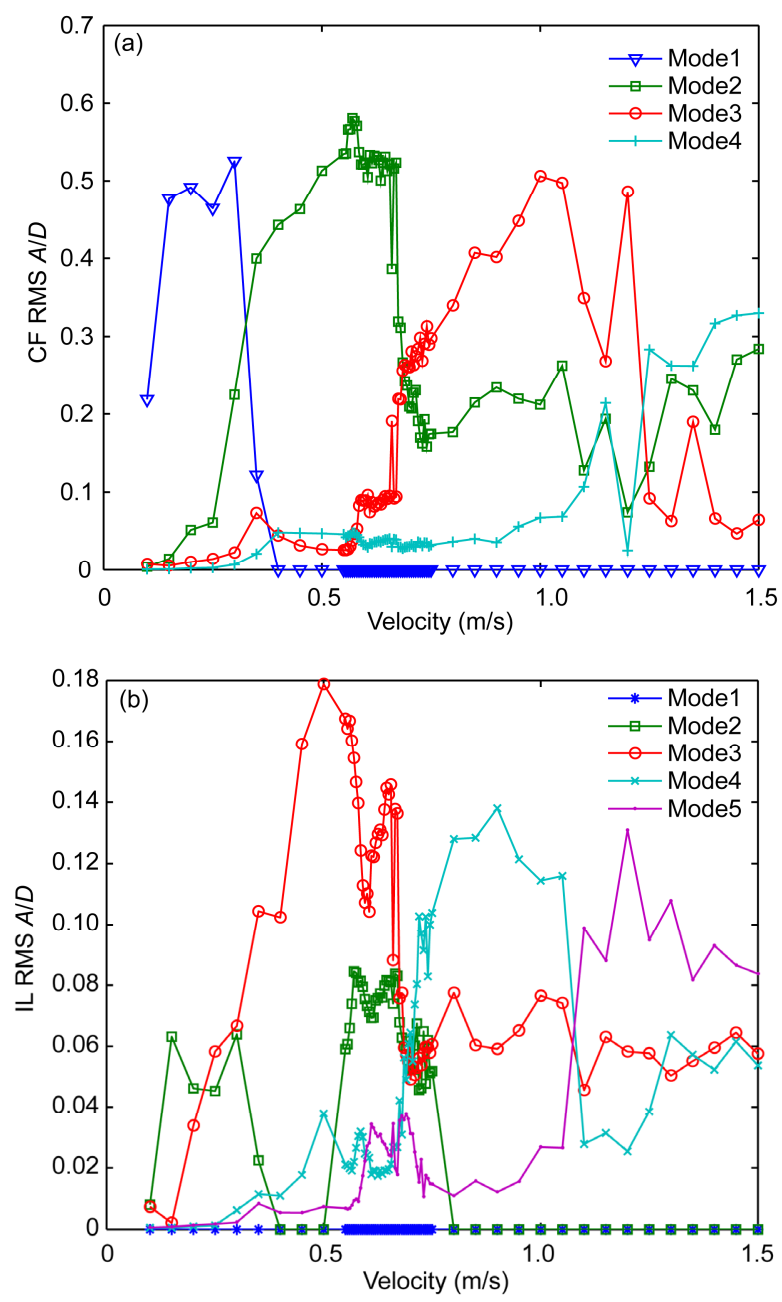

Figure 16 (Color online) (a) CF and (b) IL Maximum RMS modal displacement amplitudes along the length.

Table 4 Competing modes in non-resonant regions

\begin{tabular}{ccc}
\hline Region & CF modes & IL modes \\
\hline Non-resonant region 1 & 1,2 & 2,3 \\
Non-resonant region 2 & 2,3 & $2,3,4$ \\
Non-resonant region 3 & $2,3,4$ & $3,4,5$ \\
\hline
\end{tabular}


slender cylinder. The experimental results show that, when dual resonance occurs, the CF and IL displacements exhibit regular harmonic responses. Steady motion orbits and phase angle differences were also observed along the length of the model. The variations of strain, displacement and the dominant frequency with the towing speed are presented.

For each test in non-resonant regions, the motion orbit shapes are quite messy without any steady phase angle difference prevailing at all measured points along the model. The spectra of the displacement or strain at most of the locations exhibit more than one peak, indicating that more than one mode participate in the vibrations of the model. In non-resonant regions, the mode order number changes, and the dominant frequency of strain experiences suddenly rises. The CF and IL modal displacements indicate that mode competition is the dominant factor in the non-resonant regions compared to that in the resonant regions, and thus no one mode can prevail over other modes. It may be inferred that due to the competition, some vibration energy dissipates, resulting in a lower displacement amplitude in non-resonant regions.

This work was supported by the Knowledge Innovation Program of Chinese Academy of Sciences (Grant No. KJCX2-YW-L07) and Construction Technology Program of Ministry of Transport (Grant No. 2013318740 050).

1 Sarpkaya T. A critical review of the intrinsic nature of vortex-induced vibrations. J Fluids Struct, 2004, 19: 389-447

2 Williamson C H K, Govardhan R. Vortex-induced vibrations. Annu Rev Fluid Mech, 2004, 36: 413-455

3 Gabbai R D, Benaroya H. An overview of modeling and experiments of vortex-induced vibration of circular cylinders. J Sound Vib, 2005, 282: 575-616

4 Ge F, Long X, Wang L, et al. Flow-induced vibrations of long circular cylinders modeled by coupled nonlinear oscillators. Sci China Ser G-Phys Mech Astron, 2009, 52(7): 1086-1093

5 Zhang M M, Xu J Z. Active control of fluctuating pressure induced by blade-vortex interaction. Sci China-Tech Sci, 2011, 54(4): 862-868

6 Wang Y K, Shan J X, Tian W, at al. Mechanism of perturbation-combined active control technique for asymmetric vortex flow over slender body at high angle of attack. Sci China-Tech Sci, 2011, 54(10): 2665-2673
7 Zhang H, Fan B C, Li H Z. Suppression of vortex-induced vibration of a circular cylinder by Lorentz force. Sci China-Phys Mech Astron, 2011, 54(12): 2248-2259

8 Chu H B, Zhang B Q, Chen Y C, at al. Investigation of micro vortex generators on controlling flow separation over SCCH high-lift configuration. Sci China-Tech Sci, 2012, 55(7): 1943-1953

9 Wu X D, Ge F, Hong Y S. A review of recent studies on vortex-induced vibrations of long slender cylinders. J Fluids Struct, 2012, 28: 292-308

10 Chaplin J R, Bearman P W, Huera-Huarte F J, et al. Laboratory measurements of vortex-induced vibrations of a vertical tension riser in a stepped current. J Fluids Struct, 2005, 21: 3-24

11 Trim A D, Braaten H, Lie H, et al. Experimental investigation of vortex-induced vibration of long marine risers. J Fluids Struct, 2005, 21: 335-361

12 Baarholm G S, Larsen C M, Lie H. On fatigue damage accumulation from in-line and cross-flow vortex-induced vibrations on risers. J Fluids Struct, 2006, 22: 109-127

13 Lie H, Kaasen K E. Modal analysis of measurements from a large-scale VIV model test of a riser in linearly sheared flow. J Fluids Struct, 2006, 22: 557-575

14 Song J N, Lu L, Teng B, et al. Laboratory tests of vortex-induced vibrations of a long slender riser pipe subjected to uniform flow. Ocean Eng, 2011, 38: 1308-1322

15 Li X C, Wang Y X, Li G W, et al. Experimental investigation of vortex-induced vibrations of long free spans near seabed. Sci China-Tech Sci, 2011, 54(3): 698-704

16 Tang G Q, Lu L, Teng B, et al. Identification of hydrodynamic coefficients from experiment of vortex-induced vibration of slender riser model. Sci China-Tech Sci, 2011, 54(7): 1894-1905

17 Ma L Q, Feng L H. Experimental investigation on control of vortex shedding mode of a circular cylinder using synthetic jets placed at stagnation points. Sci China-Tech Sci, 2013, 56(1): 158-170

18 Blackburn H M, Henderson R D. Lock-in behavior in simulated vortex-induced vibration. Exp Thermal Fluid Sci, 1996, 12: 184-189

19 de Langre E. Frequency lock-in is caused by coupled-mode flutter. J Fluids Struct, 2006, 22: 783-791

20 Dahl J M, Hover F S, Triantafyllou M S. Dual resonance in vortex-induced vibrations at subcritical and supercritical Reynolds numbers. J Fluid Mech, 2010, 643: 395-424

21 Vandiver J K, Jaiswal V, Jhingran V. Insights on vortex-induced, traveling waves on long risers. J Fluids Struct, 2009, 25: 641-653

22 Khalak A, Williamson C H K. Motions, forces and mode transitions in vortex-induced vibrations at low mass-damping. J Fluids Struct, 1999, 13: 813-851

23 Tass P, Rosenblum M G, Weule J, et al. Detection of n:m phase locking from noisy data: Application to magnetoencephalography. Phys Rev Lett, 1998, 81: 3291-3294

24 Huera-Huarte F J, Bearman P W. Wake structures and vortex-induced vibrations of along slender cylinder-Part 1: Dynamic response. J Fluids Struct, 2009, 25: 969-990 